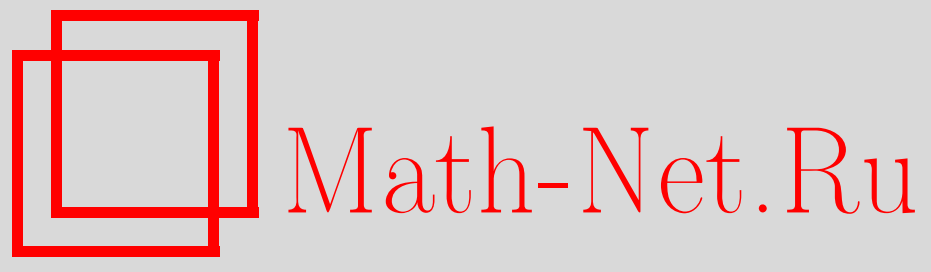

Д. М. Ушанов, Теорема Быковского и обобщение теоремы Ларчера, Матем. заметки, 2012, том 91, выпуск $5,795-798$

DOI: https://doi.org/10.4213/mzm9366

Использование Общероссийского математического портала Math-Net.Ru подразумевает, что вы прочитали и согласны с пользовательским соглашением http://www . mathnet.ru/rus/agreement

Параметры загрузки:

IP : 54.197 .130 .99

26 апреля 2023 г., 13:49:31

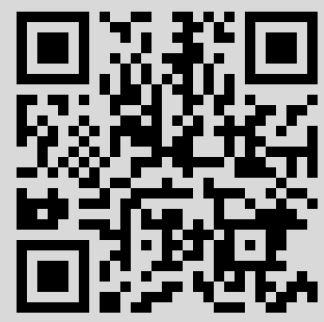




\section{Теорема Быковского и обобщение теоремы Ларчера}

\section{Д. М. Ушанов}

1. Введение. Пусть $a=\left(a_{1}, \ldots, a_{s}\right)$ - произвольный набор целых чисел, а $N \geqslant 3$ натуральное число. Сеткой Коробова (см. [1]) называется множество

$$
\mathscr{K}(a)=\left\{\left(\left\{\frac{a_{1} k}{N}\right\}, \ldots,\left\{\frac{a_{s} k}{N}\right\}\right) \mid k=1,2, \ldots, N\right\} .
$$

Рассмотрим решетку

$$
\Gamma_{N}(a)=\left\{\left(m_{1}, \ldots, m_{s}\right) \in \mathbb{Z}^{s} \mid a_{1} m_{1}+\cdots+a_{s} m_{s} \equiv 0(\bmod N)\right\} ;
$$

несложно проверить, что

$$
\mathscr{K}(a)=\Gamma_{N}^{*}(a) \cap[0,1)^{s},
$$

где $\Gamma_{N}^{*}(a)$ - двойственная к $\Gamma_{N}(a)$ решетка.

Для последовательности $\Xi=\left\{\xi_{x} \in[0,1]^{s}, x=0,1,2, \ldots, N-1\right\}$ отклонением называется следующая величина:

$$
D(\Xi)=\sup _{\gamma_{1}, \ldots \gamma_{s} \in[0,1]}\left|\#\left\{x: 0 \leqslant x<N, \xi_{x} \in\left[0, \gamma_{1}\right) \times \cdots \times\left[0, \gamma_{s}\right)\right\}-\gamma_{1} \cdots \gamma_{s} N\right| .
$$

В [2] Ларчер получил следующий результат.

Теорема А. Существует постоянная с такая, что для любого натурального $N$ существует натуральное $g,(g, N)=1$ и отклонение сетки $\mathscr{K}(1, g)$ оченивается

$$
D_{N}(\mathscr{K}(1, g))<c \frac{N \log N \log \log N}{\phi(N)} .
$$

Доказательство теоремы элементарно и опирается на аппарат цепных дробей.

Недавно Быковский получил следующий результат (см. [3], [4]).

Теорема В. Для любого натурального $s \geqslant 2$ существует постоянная с такая, что для любого натурального $N$ найдется такой набор $\left(a_{1}, \ldots, a_{s}\right)$, что отклонение сетки $\mathscr{K}\left(a_{1}, \ldots, a_{s}\right)$ оценивается

$$
D_{N}\left(\mathscr{K}\left(a_{1}, \ldots, a_{s}\right)\right)<c \log ^{s-1} N \log \log N .
$$

Доказательство этой теоремы использует аналитический аппарат.

Ларчер получил также следующий результат для вычетов степени $k$ (см. $[2 ;$ следствие 5]). Для любого $k \in \mathbb{N}$ существует константа $c(k)$ такая, что для любого $N \in \mathbb{N}$ найдется $x \in \mathbb{N},(x, N)=1,1 \leqslant x<N$, такой что существует $x_{1}, x \equiv x_{1}^{k}(\bmod N)$, и для разложения в цепную дробь $x / N=\left[0 ; b_{1}, \ldots, b_{l}\right]$ выполнено

$$
\sum b_{i}<c(k)(\log N \log \log N)^{2} .
$$

Отсюда следует (см. Островский [5]) оценка на отклонение двумерной последовательности: для такого $x$ будет верно

$$
D(\mathscr{K}(1, x)) \ll(\log N \log \log N)^{2} .
$$

В работе [6] получено усиление теоремы Ларчера на случай, когда вместо множества вычетов фиксированной степени при простом $N$ рассматривается произвольная достаточно большая мультипликативная подгруппа группы $\mathbb{Z}_{p}^{*}$.

Работа выполнена при поддержке Российского фонда фундаментальных исследований (грант № 12-01-00681).

(C) Д. М. УШАнов, 2012 
Теорема С. Пусть р простое, $G$ - мультипликативная подгруппа в $\mathbb{Z}_{p}^{*}$. Пусть

$$
\# G \geqslant 10^{8} p^{7 / 8} \log ^{5 / 2} p .
$$

Тогда существует элемент $a \in G, a / p=\left[0 ; b_{1}, b_{2}, \ldots, b_{l}\right], c$

$$
D(\mathscr{K}(1, a)) \ll \sum_{i=1}^{l} b_{i} \leqslant 500 \log p \log \log p .
$$

Отметим, что недавно Чанг независимо получила теорему С (см. [7]).

Пользуясь результатами Быковского (см. [3], [4]), в настоящей работе мы обобщили результат Ларчера на большие размерности и получили следующую теорему.

Tеорема 1. Обозначим

$$
\alpha_{m}=\frac{2^{m-1}}{2^{m+1}-m-2}, \quad \beta_{m}=\frac{2^{m}}{2^{m+2}-m-4}, \quad n_{\delta}=\max \left(3,\left\lceil\frac{81}{\delta}+\frac{1}{\ln 2}-160\right\rceil\right) .
$$

Определим функиии $s^{\prime}(\delta)$ и $s^{\prime \prime}(\delta)$ следующим образом:

$$
\begin{aligned}
& s^{\prime}(\delta)=\left\{\begin{array}{l}
\left\lceil\frac{2 m^{2}(\delta-1)}{4 \delta\left(2^{-m}-1\right)+1}\right]+2, \quad \text { если } \beta_{m} \leqslant \delta<\alpha_{m}, \\
\left\lceil\frac{2 m(\delta-1)(m+1)}{\delta\left(3 \cdot 2^{-m}-4\right)+1}\right\rceil+2, \quad \text { eсли } \alpha_{m+1} \leqslant \delta<\beta_{m},
\end{array}\right. \\
& s^{\prime \prime}(\delta)=\left\lfloor\frac{(1-\delta) 2^{n_{\delta}}}{\left(\delta\left(160+n_{\delta}\right) / 81-1\right) \cdot 0.45}\right\rfloor+3 .
\end{aligned}
$$

Пусть $s_{\min }(\delta)=\min \left(s^{\prime}(\delta), s^{\prime \prime}(\delta)\right)$.

Если $s \geqslant 3$ и $\delta \in(0,1)$ таковы, что $s \geqslant s_{\min }(\delta)$, то для любого простого $p \geqslant 3$, любой подгруппы $G$ мультипликативной группъ $\mathbb{Z}_{p}^{*}$, такой, что $\# G \geqslant p^{\delta}$, найдутся элементь $a_{1}, \ldots, a_{s} \in G$, для которых верно

$$
D_{p}\left(\mathcal{K}\left(a_{1}, \ldots, a_{s}\right)\right) \ll \delta, s \log ^{s-1} p \log \log p .
$$

Подставляя несколько первых значений $m$ в формулировке теоремы 1 , получим удобное для анализа следствие.

Следствие 1. Пусть s и $\delta$ таковы, что

$$
s \geqslant\left\{\begin{array} { l l l } 
{ 3 , } & { \delta \in [ \frac { 3 } { 4 } , 1 ) , } \\
{ 4 , } & { \delta \in [ \frac { 2 } { 3 } , \frac { 3 } { 4 } ) , } \\
{ 5 , } & { \delta \in [ \frac { 1 4 } { 2 3 } , \frac { 2 } { 3 } ) , } \\
{ 6 , } & { \delta \in [ \frac { 4 } { 7 } , \frac { 1 4 } { 2 3 } ) , } \\
{ 7 , } & { \delta \in [ \frac { 6 } { 1 1 } , \frac { 4 } { 7 } ) , } \\
{ 8 , } & { \delta \in [ \frac { 1 0 } { 1 9 } , \frac { 6 } { 1 1 } ) , } \\
{ 9 , } & { \delta \in [ \frac { 2 2 } { 4 3 } , \frac { 1 0 } { 1 9 } ) , } \\
{ 1 0 , } & { \delta \in [ \frac { 1 } { 2 } , \frac { 2 2 } { 4 3 } ) , } \\
{ 1 1 , } & { \delta \in [ \frac { 1 7 } { 3 5 } , \frac { 1 } { 2 } ) , }
\end{array} \quad \left\{\begin{array}{ll}
13, & \delta \in\left[\frac{9}{41}, \frac{17}{35}, \frac{9}{19}\right), \\
15, & \delta \in\left[\frac{19}{41}\right), \\
17, & \delta \in\left[\frac{23}{25}, \frac{11}{53}, \frac{21}{25}\right), \\
18, & \delta \in\left[\frac{3}{7}, \frac{23}{53}\right), \\
19, & \delta \in\left[\frac{25}{59}, \frac{3}{7}\right), \\
20, & \delta \in\left[\frac{13}{31}, \frac{25}{59}\right) .
\end{array}\right.\right.
$$


Тогда для любого простого $p \geqslant 3$, любой подгруппы $G$ мулътипликативной группы $\mathbb{Z}_{p}^{*}$, такой, что $\# G \geqslant p^{\delta}$, найдутся элементы $a_{1}, \ldots, a_{s} \in G$, для которых верно

$$
D_{p}\left(\mathcal{K}\left(a_{1}, \ldots, a_{s}\right)\right) \ll \delta, s \log ^{s-1} p \log \log p .
$$

2. Основые идеи доказательства. Всюду далее мы будем использовать следующие обозначения. Пусть $G$ - подгруппа мультипликативной группы $\mathbb{Z}_{p}^{*}$ чисел по модулю $p$. Обозначим через $A\left(P_{1}, \ldots, P_{s}\right)$ число решений сравнения

$$
a_{1} m_{1}+\cdots+a_{s} m_{s} \equiv 0(\bmod N)
$$

в целых $a_{1}, \ldots, a_{s} \in G$ и целых $m_{1}, \ldots, m_{s}$, таких, что

$$
1 \leqslant m_{1} \leqslant P_{1}, \ldots, 1 \leqslant m_{s} \leqslant P_{s} .
$$

Лемма 1. Пусть параметры s и $\delta$ выбраны так, как в теореме 1. Тогда

$$
A\left(P_{1}, \ldots, P_{s}\right) \ll \frac{\# G^{s}}{p} P_{1} \cdots P_{s} .
$$

ДоказАтельство. Обозначим $e_{p}(t)=\exp (2 \pi i t / p)$,

$$
S(t, G)=\sum_{g \in G} e_{p}(t g), \quad S(G)=\max _{t \neq 0}|S(t, G)| .
$$

Сначала рассмотрим случай, когда $s \geqslant s^{\prime}(\delta)$.

Заметим, что количество решений сравнения равно

$$
A\left(P_{1}, \ldots, P_{s}\right)=\frac{1}{p} \sum_{1 \leqslant m_{i} \leqslant P_{i}} \sum_{a_{i} \in G} \sum_{n=1}^{p} e_{p}\left(n\left(a_{1} m_{1}+\cdots+a_{s} m_{s}\right)\right) .
$$

Воспользовавшись неравенством Коши-Буняковского, получим

$$
A\left(P_{1}, \ldots, P_{s}\right) \leqslant \frac{1}{p} \sum_{m} S^{s-2}(G) \cdot p \# G .
$$

Для оценки $S(G)$ сверху мы воспользуемся результатом Конягина (см. [8]). А именно, в обозначениях формулировки теоремы 1

- если существует такое $m \in \mathbb{N}$, что $\beta_{m} \leqslant \delta<\alpha_{m}$, то

$$
S(G) \ll_{m} \# G^{1-2 / m^{2}+1 / 2^{m-1} m^{2}} p^{1 / 2 m^{2}},
$$

- если же существует такое $m \in \mathbb{N}$, что $\alpha_{m+1} \leqslant \delta<\beta_{m}$, то

$$
S(G) \ll_{m} \# G^{1-2 / m(m+1)+3 / 2^{m+1} m(m+1)} p^{1 / 2 m(m+1)} .
$$

Подставляя оценки на $S(G)$ в оценку (1) для $A\left(P_{1}, \ldots, P_{s}\right)$, мы получим

$$
A\left(P_{1}, \ldots, P_{s}\right) \leqslant \frac{\# G^{s}}{p} P_{1} \cdots P_{s} .
$$

В случае $s \geqslant s^{\prime \prime}(\delta)$ мы рассуждаем так же, разница заключается в другой оценке $S(G)$.

Мы используем следующий результат Гараева (см. [9; теорема 4.1]). Если $3 \leqslant n \leqslant$ $1.44 \log \log p$ - натуральное число, $c>0$ - произвольная постоянная, $X_{1}, \ldots, X_{n}$ - подмножества $Z_{p}^{*}$, удовлетворяющие условию

$$
\# X_{1} \cdot \# X_{2} \cdot\left(\# X_{3} \cdots \# X_{n}\right)^{1 / 81}>p^{1+c},
$$

то имеет место оценка

$$
\left|\sum_{x_{1} \in X_{1}} \cdots \sum_{x_{n} \in X_{n}} e_{p}\left(x_{1} \cdots x_{n}\right)\right| \ll \# X_{1} \cdots \# X_{n} p^{-0.45 c / 2^{n}} .
$$

Взяв $X_{1}=t G, X_{2}=G, \ldots, X_{n}=G$, и подобрав подходящим образом параметры $c$ и $n$, мы получим лемму. 
3. Схема доказательства теоремы 1. Выберем параметр $Q=p /\left(2 \log ^{s+1} p\right)$. Пусть $a=\left(a_{1}, \ldots, a_{s}\right)$. Для целочисленного вектора $m=\left(m_{1}, \ldots, m_{s}\right)$ определим

$$
H(m)=\max \left(1,\left|m_{1}\right|\right) \cdots \max \left(1,\left|m_{s}\right|\right) .
$$

Определим функцию

$$
q(a)=\min \left\{H(m) \mid a_{1} m_{1}+\cdots+a_{s} m_{s} \equiv 0(\bmod N)\right\} .
$$

Определим множества $\Omega_{1}$ и $\Omega$ следующим образом:

$$
\Omega_{1}=\left\{a \in G^{s} \mid q(a) \leqslant Q\right\}, \quad \Omega=\left\{a \in G^{s} \mid q(a)>Q\right\} .
$$

Очевидно, что \#( $\left.\Omega_{1} \cup \Omega\right)=\# G^{s}$. С помощью леммы 1 мы показываем, что \# $\Omega_{1} \leqslant$ $\# G^{s} / 2$; следовательно, \# $\#>\# G^{s} / 2$.

Ненулевой узел $\gamma$ решетки Г называется относительным минимумом, если не найдется другого ненулевого узла $\gamma^{\prime}$ из $Г$, для которого

$$
\left|\gamma_{1}^{\prime}\right| \leqslant\left|\gamma_{1}\right|, \quad \ldots, \quad\left|\gamma_{s}^{\prime}\right| \leqslant\left|\gamma_{s}\right| .
$$

Обозначим через $\mathfrak{M}(\Gamma)$ множество всех относительных минимумов решетки $Г$.

Далее мы воспользуемся следующим результатом Быковского (см. [4]): для любой сетки Коробова верно неравенство

$$
D_{N}(\mathscr{K}(a)) \ll N \sum_{m \in \mathfrak{M}\left(\Gamma_{N}(a)\right)} \frac{1}{H(m)} .
$$

Таким образом, усредняя по множеству $\# \Omega$, получим

$$
\min _{a \in G^{s}} D(\mathscr{K}(a)) \ll \frac{p}{\# \Omega} \sum_{a \in G^{s}} \sum_{Q \leqslant H(m) \leqslant p} \frac{\delta_{p}\left(a_{1} m_{1}+\cdots+a_{s} m_{s}\right)}{H(m)} .
$$

Оценивая последнюю сумму, получаем теорему.

Автор выражает благодарность И. Шпарлинскому за указание на возможность улучшения результата.

\section{СПИСОК ЦИТИРОВАННОЙ ЛИТЕРАТУРЫ}

[1] H. М. Коробов, УМН, 22:3 (1967), 83-118. [2] G. Larcher, Monatsh. Math., 101:2 (1986), 135-150. [3] V. A. Bykovskii, 27th Journées Arithmétiques, Program and Abstract Book, Vilnius University, 2011, 10-11. [4] В. А. Быковский, "Отклонение сеток Коробова", Изв. PAH. Сер. матем. (в печати). [5] A. Ostrowski, Abh. Math. Sem. Hamburg, 1 (1922), 77-98; 249-250. [6] N. G. Moshchevitin, D. M. Ushanov, Unif. Distrib. Theory, 5:1 (2010), 45-52. [7] M.-C. Chang, C. R. Math. Acad. Sci. Paris, 349:13-14 (2011), 713-718. [8] C. В. Конягин, IV Межденародная конференция "Современные проблемы теории чисел и ее приложения". Актуалъные проблемы, Часть 3, М., Мех.-мат. фак-т МГУ, 2002, 86-114. [9] М. З. Гараев, УМH, 65:4 (2010), 5-66.

\section{Д. М. Ушанов}

